\title{
All-Optical Switching Based on the Plasma Channel Induced by Laser Pulses
}

\author{
Chao Tan (D), Binliang Hu ${ }^{D}$, Shiping Zhan, Yonghua Hu, and Bin Zhong \\ School of Information and Electrical Engineering, Hunan University of Science and Technology, Xiangtan 411201, China \\ Correspondence should be addressed to Chao Tan; chaotanhnu@163.com and Binliang Hu; blhu@hnust.edu.cn
}

Received 19 June 2018; Revised 20 August 2018; Accepted 29 August 2018; Published 1 October 2018

Academic Editor: Xinxing Zhou

Copyright (c) 2018 Chao Tan et al. This is an open access article distributed under the Creative Commons Attribution License, which permits unrestricted use, distribution, and reproduction in any medium, provided the original work is properly cited.

\begin{abstract}
We display a theoretical and experimental study of all-optical switching for signal lasers based on the plasma channel induced by the control laser. Using the plasma channel generated in the carbon disulfide $\left(\mathrm{CS}_{2}\right)$ solution, the signal light can be modulated as some spatial distributions including unchanging, ring-shaped beam, and other intensity profiles. The modulation on the signal light can be conveniently adjusted by changing the control light's incident intensity distribution. We can infer the dark spot shape in the modulated signal laser through the intensity profile of control laser beam. These results provide the great potential of plasma channel induced by lasers as an all-optical switching for various optoelectronic applications.
\end{abstract}

\section{Introduction}

All-optical switching is one of the important components of all-optical signal processing, which aims towards high-speed optical communications $[1,2]$ and high-performance optical computing [3-5]. Owing to special properties of controlling light propagation, photonic crystals are thought to have promising future in all-optical switching. Two photonic crystal (PC) cavities, metallic PC, and electromagnetically induced transparency in PC nanocavities $[6,7]$ are reported to use for all-optical switching. Thanks to strong localized field enhancement effect, high localization, and the breakthrough of the diffraction limit, surface plasmon is chosen to realize ultrafast all-optical switching with few energy consumption. In the 2016, Chai et al. successfully designed an ultrafast all-optical switch using plasma-photon-hybrid nanostructures-coated multicomponent nanocomposite [8]. Neira et al. propose a general, easy-to-implement scheme for all-optical switch using epsilon-near-zero (ENZ) materials $[9,10]$. Microring resonators are also generally considered as an ideal platform for demonstrating all-optical switching $[11,12]$. Various structures in metamaterials also provide ideas for achieving all-optical switching with high performance. In designs of switchable devices, ultrafast all-optical switching has been achieved based on silicon, crystalline Ge, gallium arsenide, and ErAs/GaAs superlattices [13-16].
Using phase-change materials (such as vanadium dioxide, germanium antimony telluride and $\mathrm{Ge}_{2} \mathrm{Sb}_{2} \mathrm{Te}_{5}$ ), the highspeed all-optical modulation on the signal laser is reported [17-19]. Ellis et al. explore two mechanisms for all-optical switching in ferromagnets [20], modeling ultrafast all-optical switching in synthetic ferrimagnets is then demonstrated by Gerlach et al. [21]. Graphene, topological insulators, transition metal dichalcogenide, and other 2D materials [2224 ] are proved to be superb candidates for creating novel ultrafast all-optical switching devices.

When laser pulses propagate in a nonlinear media, plasma channels are generated by ionization. The electron density distribution and refractive index distribution of the plasma are nonuniform. According to Fermat's principle, the beam changes its direction of propagation in the plasma channel. Previously, we used the plasma channel to plasma optical modulation for laser beams $[25,26]$. Naturally, an idea is shown that this plasma can be an excellent nonlinear medium for realizing all-optical switching.

In this paper, firstly, we theoretically investigate all-optical switching for signal lasers based on the plasma channel. Then, the dynamic experiment is performed by keeping the control light and signal light copropagating through $\mathrm{CS}_{2}$. We observe the modulation on the signal laser beam induced by plasma channel in $\mathrm{CS}_{2}$. It is uncovered that the signal light can be modulated as some spatial intensity distribution 
(unchanging, ring-shaped beam, and other intensity profiles) by changing the incident intensity of the control light, and the corresponding mechanism is discussed. Theoretical analysis and experimental results are consistent, which fully illustrated that this all-optical switching induced by plasma channel is reasonable.

\section{Theoretical Analysis}

We consider the propagation of a linearly polarized laser in a preformed plasma channel. The effect of the relativistic nonlinearity and the ponderomotive nonlinearity on the beam is neglected. The normalized equation for describing the propagation of laser beams is given by [27]

$$
\begin{gathered}
\left\{\nabla_{\perp}^{2}+2 i k \frac{\partial}{\partial z}+\left(1-\beta_{g}^{2}\right) \frac{\partial^{2}}{\partial \zeta^{2}}\right\} \psi(\vec{r}, z, \zeta) \\
=\frac{\omega_{p}^{2}(\vec{r})}{c^{2}} \psi(\vec{r}, z, \zeta)
\end{gathered}
$$

where $\beta_{g}, \psi(\vec{r}, z, t)$, and $\omega_{p}(\vec{r})$ are the normalized group velocity, normalized electric field amplitude, and plasma frequency, respectively.

The modified approximate solution of amplitude can be derived by Wentzel-Kramers-Brillouin-Jeffreys (WKBJ) approximation [25].

$$
\begin{aligned}
A(x, y, z)= & A(x, y, 0) \exp \left[-\frac{1}{k} \int_{0}^{z}\left(\frac{\partial A(x, y, 0)}{\partial x} \frac{\partial \varphi\left(x, y, z^{\prime}\right)}{\partial x}+\frac{\partial A(x, y, 0)}{\partial y} \frac{\partial \varphi\left(x, y, z^{\prime}\right)}{\partial y}\right) d z^{\prime}\right] \\
& \times \exp \left[-\frac{1}{2 k} \int_{0}^{z}\left(\frac{\partial^{2} \varphi\left(x, y, z^{\prime}\right)}{\partial x^{2}}+\frac{\partial^{2} \varphi\left(x, y, z^{\prime}\right)}{\partial y^{2}}\right) d z^{\prime}\right]
\end{aligned}
$$

We consider the propagation of the signal laser beam with a Gaussian profile $A_{\text {signal }}(x, y, 0)=A_{0} \exp \left[-\left(x^{2}+\right.\right.$ $\left.\left.y^{2}\right) / 2 w^{2}\right]$. The control laser also has a Gaussian intensity distribution $A_{\text {control }}(x, y, 0)=A_{0} \exp \left[-\left(\mathrm{D}_{1} x^{2}+\mathrm{D}_{2} y^{2}\right) / 2 w^{2}\right]$, where $A_{0}$ and $w$ are the initial axial amplitude and beam width, respectively. $\mathrm{D}_{1}$ and $\mathrm{D}_{2}$ are constants, the control laser has different spatial distribution under different $\mathrm{D}_{1}$ and $\mathrm{D}_{2}$. Atomic ionization degree is proportional to the light intensity of laser beams. Resulting in a plasma channel with a Gaussian electron density distribution and refractive index generated. The electron density in the plasma channel can be expressed as

$$
n_{e}(x, y, z)=n_{0} \exp \left[-\frac{\mathrm{D}_{1} x^{2}+\mathrm{D}_{2} y^{2}}{2 \sigma^{2}}\right]
$$

where $z, n_{0}$, and $\sigma$ are the propagation distance, initial axial electron density, and plasma channel width, respectively.

So, the approximate solution of amplitude equation (2) is given by

$$
\begin{aligned}
& A(x, y, z)=A_{0} \exp \left[-\frac{x^{2}+y^{2}}{2 w^{2}}+\frac{n_{0}}{n_{c}}\right. \\
& \quad \frac{\left(\mathrm{D}_{1} x^{2}+\mathrm{D}_{2} y^{2}\right) z^{2}}{2 w^{2} \sigma^{2}} \\
& \left.\cdot \exp \left(-\frac{\mathrm{D}_{1} x^{2}+\mathrm{D}_{2} y^{2}}{2 \sigma^{2}}-\frac{x^{2}+y^{2}}{2 w^{2}}\right)\right] \\
& \quad \times \exp \left\{\frac { z ^ { 2 } n _ { 0 } } { 4 n _ { c } } \left[-\frac{2}{\sigma^{2}} \exp \left(-\frac{\mathrm{D}_{1} x^{2}+\mathrm{D}_{2} y^{2}}{2 \sigma^{2}}\right)\right.\right.
\end{aligned}
$$

$$
\left.\left.+\frac{\mathrm{D}_{1} x^{2}+\mathrm{D}_{2} y^{2}}{\sigma^{4}} \exp \left(-\frac{\mathrm{D}_{1} x^{2}+\mathrm{D}_{2} y^{2}}{2 \sigma^{2}}\right)\right]\right\}
$$

where $n_{\mathrm{c}}$ is the critical plasma density.

Figure 1(a) shows spatial intensity distribution of some control laser beams with $\mathrm{D}_{1}=1, w=0.8 \mathrm{~mm}$ (half-width, at $1 / e$-intensity point). Figure 1 (b) shows spatial intensity profiles of some signal laser beams. When $\mathrm{D}_{1}=0$ and $\mathrm{D}_{2}=0$, the intensity of control laser in different spatial area is a constant. Resulting in a plasma channel with a constant electron density and refractive index generated. The signal beam is not deflected when it propagates in the plasma. So, it is easy from Figure 1(b) to see that the signal laser keep its initial intensity profile $\left[\mathrm{D}_{1}=0, \mathrm{D}_{2}=0\right]$. When the control laser propagates in a nonlinear media, the plasma channel is generated by ionization. The width, length, and initial axial electron density of the plasma are set as $0.3 \mathrm{~mm}, 5 \mathrm{~mm}$, and $0.02 n_{c}$, respectively. Based on the plasma optical modulation effect $[25,26]$, the signal laser is modulated into a ring-shaped beam by the plasma excited by the control laser $\left[D_{2}=1\right]$. As $\mathrm{D}_{2}=2$, the spatial distribution of control laser is an ellipse in shape. The dark spot in the modulated signal beam is also an ellipse in shape, the major axis of the ellipse is in the $X$ direction. As $\mathrm{D}_{2}$ increases continuously, the major axis is far longer than its minor axis. Figure 2 vividly displays the corresponding cross line $(x=0)$ of $(a)$ control lasers and (b) signal lasers when $\mathrm{D}_{2}$ is tuned. As is shown in Figure 2, the beam width of control laser decreases with the increase of $\mathrm{D}_{2}$. The light intensity of dark spot in the modulated signal laser decreases as $\mathrm{D}_{2}$ increases. The modulation on the signal laser can be conveniently adjusted by $\mathrm{D}_{2}$. We can infer the dark spot shape through the intensity profile of control laser beam. 


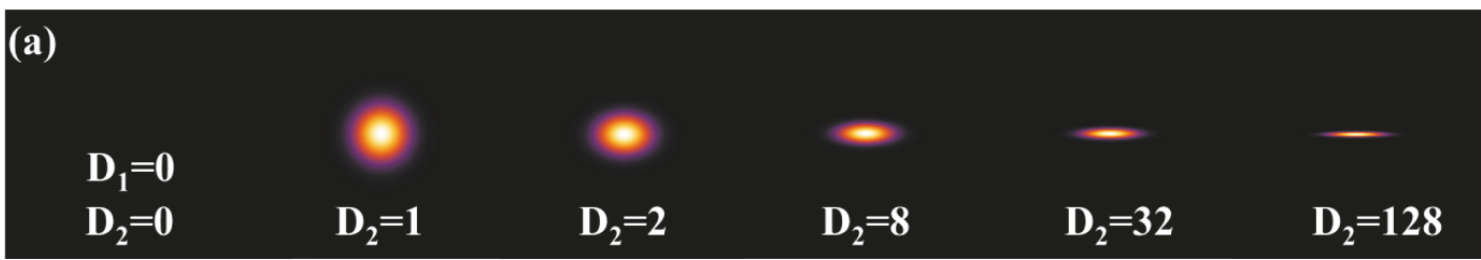

(a)

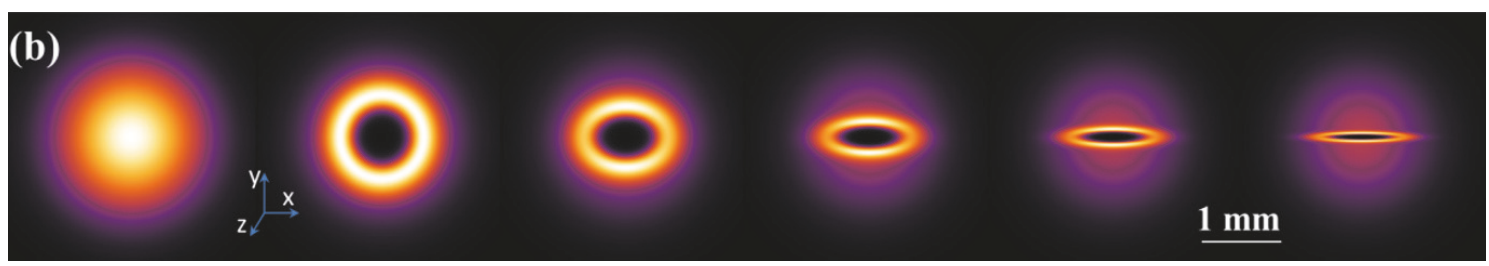

(b)

Figure 1: (a) Spatial distribution of control laser beams with $\mathrm{D}_{1}=1, \mathrm{D}_{2}=1,2,8,32$, and 128. (b) Spatial intensity profile of signal laser beams under different control beams in Figure 1(a).

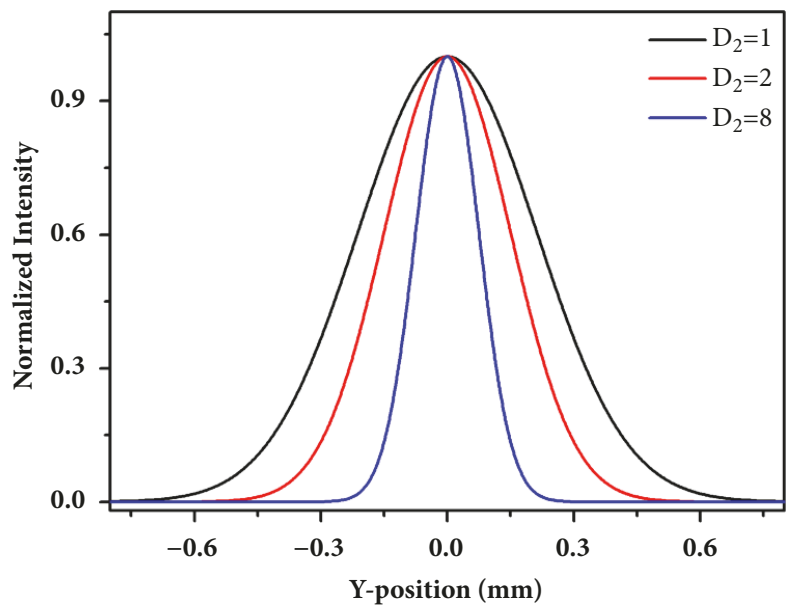

(a)

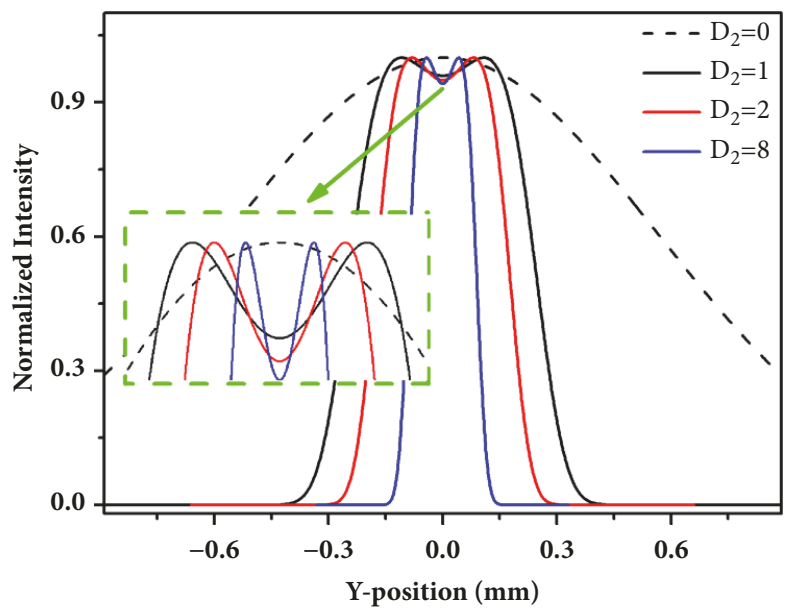

(b)

FIGURE 2: The corresponding cross line $(x=0)$ of (a) control lasers and (b) signal lasers when $\mathrm{D}_{2}$ is tuned.

Figure 3(a) shows spatial distribution of some control laser beams with $\mathrm{D}_{2}=1$. Figure $3(\mathrm{~b})$ displays the spatial profiles of signal laser beams under different control beams. When $\mathrm{D}_{1}=2$, the dark spot of modulated signal beam is an ellipse in shape, its major axis is changed into $Y$ direction. Continue to increase $\mathrm{D}_{1}$, the modulation area in the modulated signal beam increases. The energy of modulated signal laser is concentrated in the central region. These results demonstrate that the signal light can be modulated to realize some spatial distributions by changing the control light incident intensity distribution. It confirms the realization of some optical switching including unchanging, ring-shaped beam, and other intensity profiles.

\section{Experimental Results}

Experimental setup is illustrated schematically in Figure 4. All-optical switching based on the plasma channel is performed by using a femtosecond pulse laser, which is generated from an optical parametric amplifier (Libra-S, Coherent). The laser beam is pumped by a Ti:sapphire with the pulse repetition rate of $1 \mathrm{kHz}$. The Libra-S laser regenerative amplifier system contains all of the elements necessary to amplify ultrashort pulses to the $\mathrm{mJ}$ level. Elements include seed laser Vitesse, pump laser Evolution-15, optical pulse stretcher, regenerative amplifier, and optical pulse compressor. Also provided with the Libra-S is the Synchronization and Delay Generator that controls the precise timing required for regenerative amplification. Femtosecond laser pulses (control laser) are used to produce plasma channels. We use the control laser to investigate the all-optical switching based on plasma channels. He-Ne laser is used as the signal laser beam. The excitation wavelength of control laser and signal laser are $800 \mathrm{~nm}$ and $632 \mathrm{~nm}$, respectively. Intensity profiles of control and signal beams are nearly Gaussian, with beam width of $0.5 \mathrm{~mm}$ and $1.3 \mathrm{~mm}$ (full width at half maximum), 


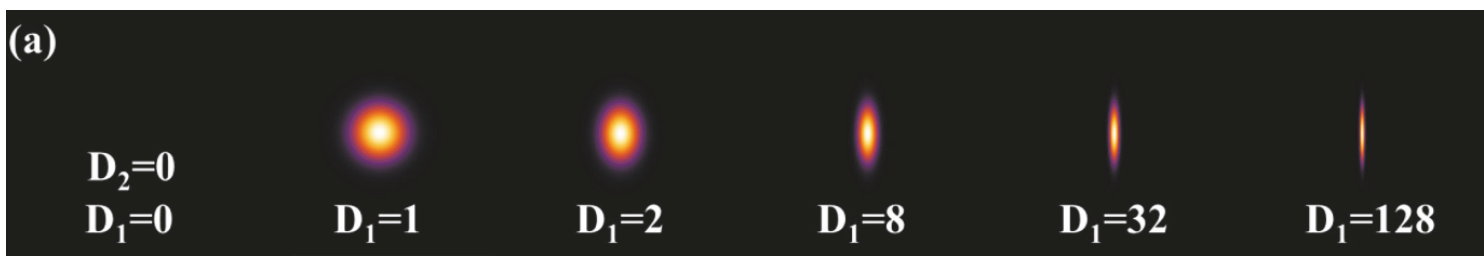

(a)

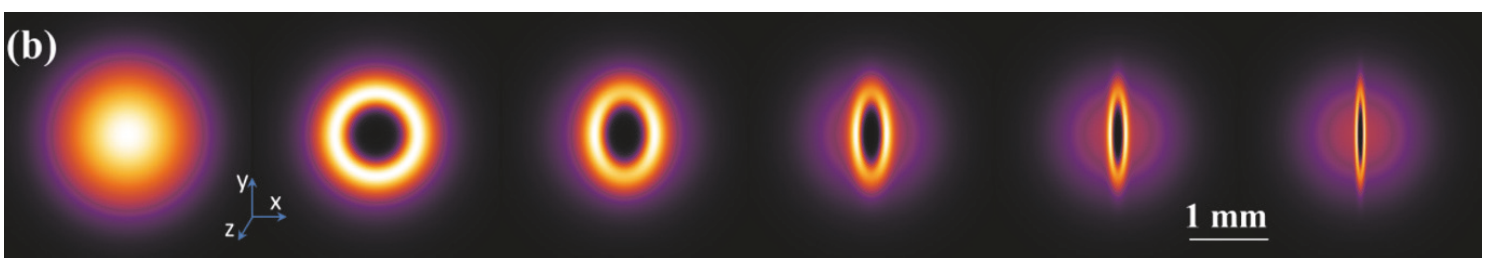

(b)

Figure 3: (a) Spatial distribution of control beams with $\mathrm{D}_{2}=1, \mathrm{D}_{1}=1,2,8,32$, and 128. (b) Spatial intensity profile of signal laser beams under different control beams in Figure 3(a).

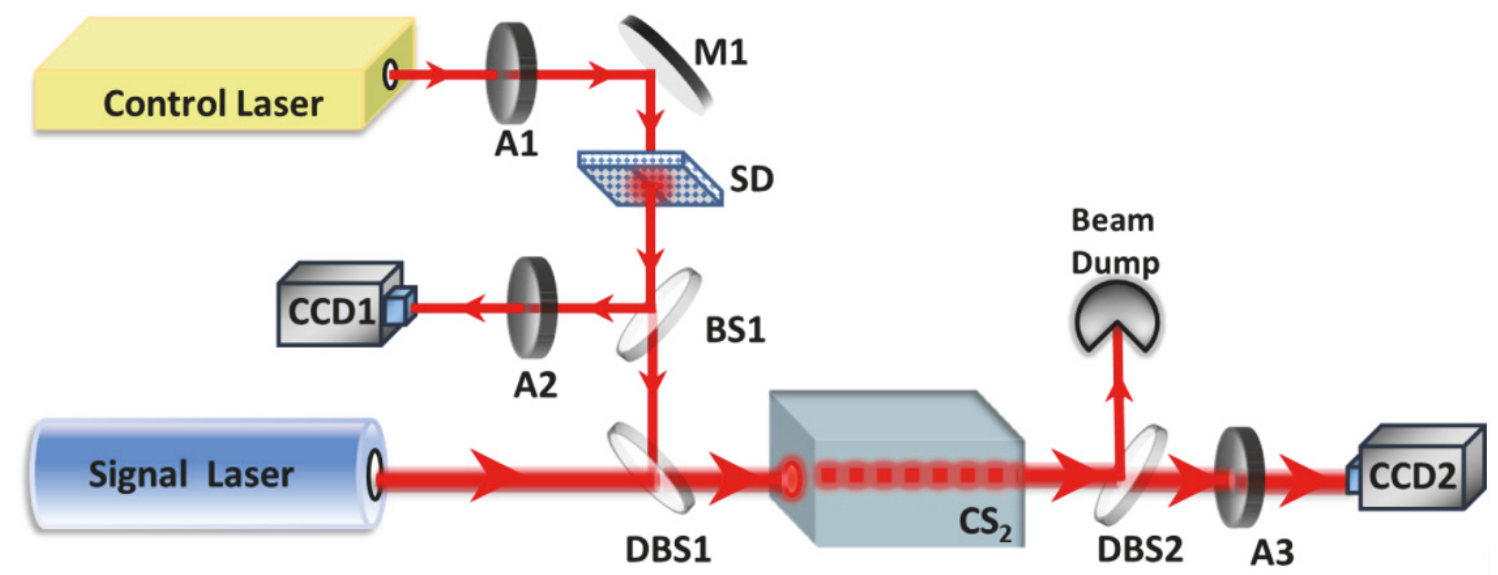

FIgURE 4: Experimental setup. A1-A3, attenuators; M1, silver-coated plane mirror; SD, beam shaping device; BS1, beam splitter; DBS1 and DBS2, dichroic beam splitter.

respectively. The intensity of control light is changed by attenuation slices. We use a beam shaping device (SD) to change the intensity distribution of the control beam. In the experiment, a cylindrical lens with focal length of $100 \mathrm{~mm}$ is used to act as SD. A high-resolution CCD camera (CCD1) is set for real-time monitoring the spatial evolution of control lasers. $\mathrm{CS}_{2}$ solution is contained in a quartz cuvette; it is used as a nonlinear media. The control light and signal light are kept copropagating through $\mathrm{CS}_{2}$. The spatial profile of the signal beam is captured and real-time monitored by a fixed CCD2 camera. The attenuators are used in front of CCD to avoid saturation and damage.

Figures 5(b) and 5(c) display spatial intensity distributions of control beams with $\mathrm{x}$-modulated and $\mathrm{y}$-modulated recorded by CCD1. Figures 5(d)-5(f) show spatial profiles of probe beams under the condition of no control laser, $\mathrm{x}$-modulated, and $\mathrm{y}$-modulated control light. Their corresponding profiles are shown in Figure 6. In the absence of control light, there is no plasma generated, the signal beam keeps its original intensity profile unchanged [Figures $5(\mathrm{a})$ and $5(\mathrm{~d})]$. When the control beam is focused by a cylindrical lens, it is easy from Figure 5(b) that its spatial intensity distribution is changed. Its spatial distribution is not strictly symmetric in the $\mathrm{X}$ and $\mathrm{Y}$ position, similar to an ellipse in shape, and its major axis is in the $\mathrm{X}$ position. Resulting in a plasma channel with an asymmetric Gaussian distribution of the electron density generated. So, an elliptic dark spot appears in the modulated signal laser beam; its major axis is in the $\mathrm{X}$ direction [Figure 5(e)]. If the control beam is focused by a cylindrical lens in the $\mathrm{Y}$ position, the intensity distribution of control laser is changed immediately. The electron density distribution of the plasma channel will also be changed. Thus, the shape of the dark spot in the signal beam is changed synchronously and the major axis of the ellipse is changed to $\mathrm{Y}$ direction [Figures 5(c) and $5(\mathrm{f})]$. If the spatial distribution of the control laser can be 


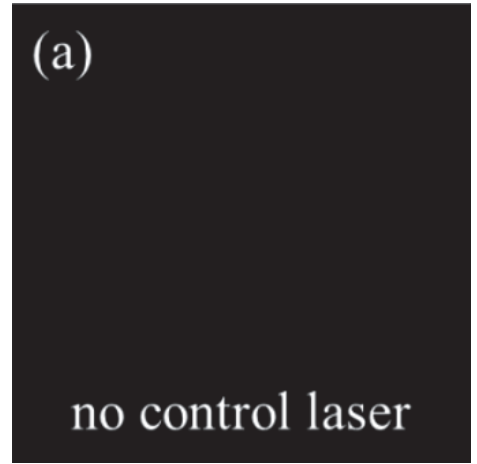

(a)

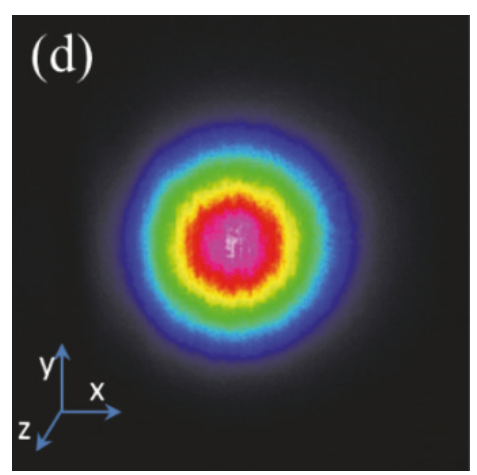

(d)

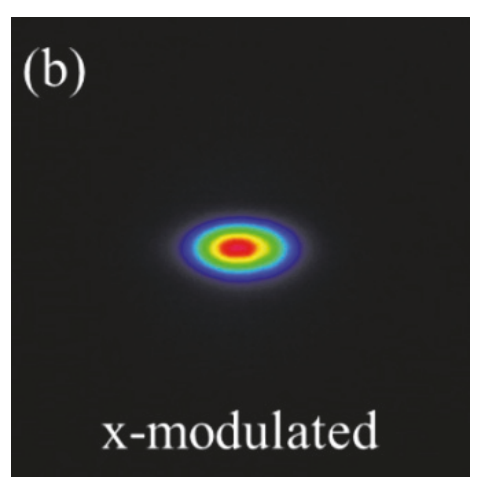

(b)

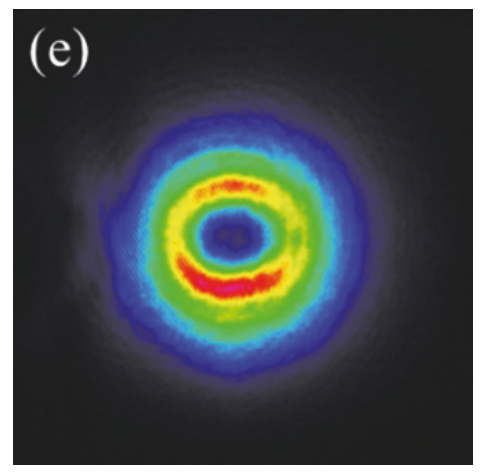

(e)

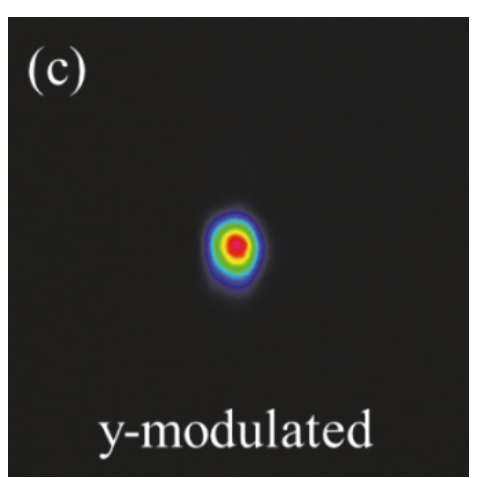

(c)

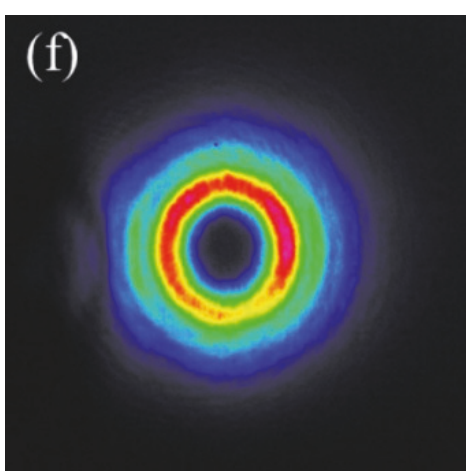

(f)

Figure 5: (b)-(c) Intensity distribution of control light with x-modulated and y-modulated. (d)-(f) Intensity profile of signal light under the condition of no control laser, $\mathrm{x}$-modulated, and $\mathrm{y}$-modulated control light.

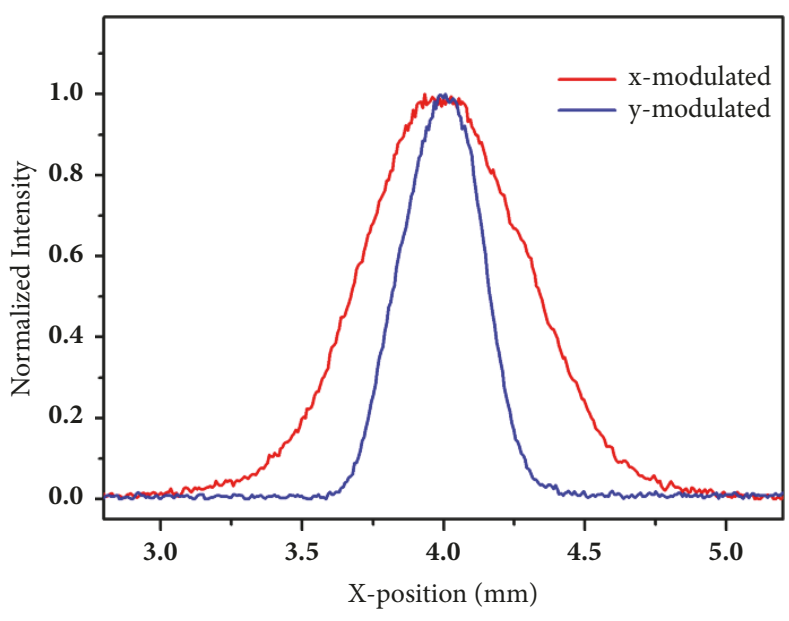

(a)

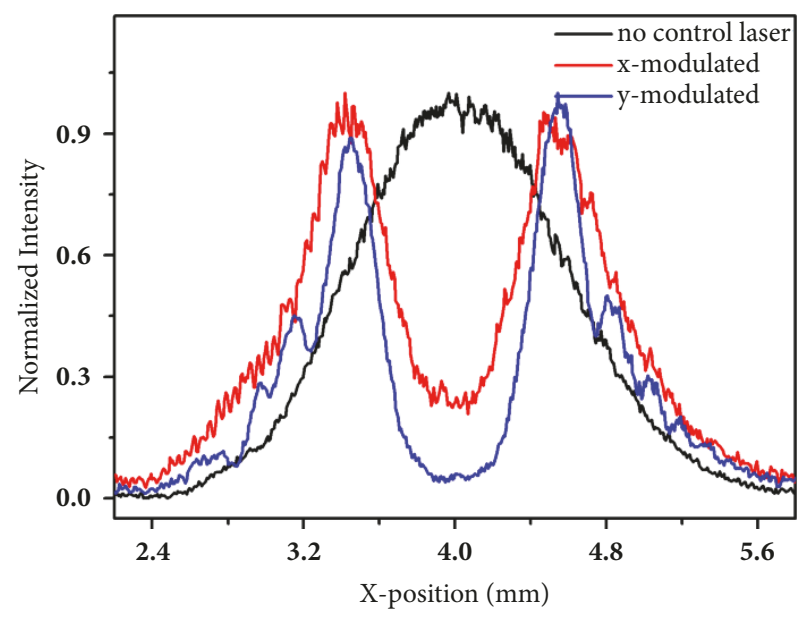

(b)

FIGURE 6: The corresponding cross line $(\mathrm{y}=0)$ of (a) control light and (b) signal light in Figure 5.

conveniently adjusted, it is expected that the signal laser beam is modulated into a laser with any spatial profile. Experimental results demonstrate the realization of some optical switching including unchanging, ring-shaped beam, and other intensity profiles. We can monitor optical switching of signal lasers dynamically by observing the spatial shape changes of the control laser.

\section{Conclusions}

In this work, we theoretically and experimentally demonstrate all-optical switching based on the plasma channel induced by femtosecond laser pulses in $\mathrm{CS}_{2}$. Our theoretical analysis is confirmed by experimental results. The signal light can be modulated by changing the control light incident 
intensity distribution. Results demonstrate the realization of some optical switching including unchanging, ring-shaped beam, and other intensity profiles. We can real-time monitor optical switching of signal lasers by observing the spatial intensity changes of the control laser.

\section{Data Availability}

The parameter and data used to support the findings of this study are included within the article.

\section{Conflicts of Interest}

The authors declare that they have no conflicts of interest.

\section{Acknowledgments}

This research was supported by National Natural Science Foundation of China (Grants nos. U1501253 and 61875054), Hunan Provincial Natural Science Foundation of China (Grants nos. 2018JJ3189 and 2017JJ3087), Scientific Research Fund of Hunan Provincial Education Department (Grants nos. 17C0624 and 15A063), and Scientific Research Startup Foundation for Doctors of Hunan University of Science and Technology (Grant no. E51662).

\section{References}

[1] A. Bazin, K. Lenglé, M. Gay et al., "Ultrafast all-optical switching and error-free $10 \mathrm{Gbit} / \mathrm{s}$ wavelength conversion in hybrid InP-silicon on insulator nanocavities using surface quantum wells," Applied Physics Letters, vol. 104, Article ID 011102, 2014.

[2] P. C. Debnath, S. Uddin, and Y.-W. Song, "Ultrafast all-optical switching incorporating in situ graphene grown along an optical fiber by the evanescent field of a laser," ACS Photonics, vol. 5, no. 2, pp. 445-455, 2018.

[3] Z. Chai, X. Hu, F. Wang, X. Niu, J. Xie, and Q. Gong, "Ultrafast All-Optical Switching," Advanced Optical Materials, vol. 5, no. 7, p. 1600665, 2017.

[4] J. S. Pelc, K. Rivoire, S. Vo, C. Santori, D. A. Fattal, and R. G. Beausoleil, "Picosecond all-optical switching in hydrogenated amorphous silicon microring resonators," Optics Express, vol. 22, no. 4, pp. 3797-3810, 2014.

[5] B. Born, J. D. A. Krupa, S. Geoffroy-Gagnon, and J. F. Holzman, "Integration of photonic nanojets and semiconductor nanoparticles for enhanced all-optical switching," Nature Communications, vol. 6, article 8097, 2015.

[6] Y. Zhao, C. Qian, K. Qiu, Y. Gao, and X. Xu, "Ultrafast optical switching using photonic molecules in photonic crystal waveguides," Optics Express, vol. 23, no. 7, pp. 9211-9220, 2015.

[7] F. Bleckmann, E. Maibach, S. Cordes, T. E. Umbach, K. Meerholz, and S. Linden, "Photochromic switching of fano resonances in metallic photonic crystal slabs," Advanced Optical Materials, vol. 2, no. 9, pp. 861-865, 2014.

[8] Z. Chai, Y. Zhu, X. Hu et al., "On-chip optical switch based on plasmon-photon hybrid nanostructure-coated multicomponent nanocomposite," Advanced Optical Materials, vol. 4, no. 8, pp. 1159-1166, 2016.
[9] A. D. Neira, G. A. Wurtz, and A. V. Zayats, "All-optical switching in silicon photonic waveguides with an epsilon-nearzero resonant cavity [Invited]," Photonics Research, vol. 6, no. 5, p. B1, 2018.

[10] T. Y. Kim, M. A. Badsha, J. Yoon, S. Y. Lee, Y. C. Jun, and C. K. Hwangbo, "General strategy for broadband coherent perfect absorption and multi-wavelength all-optical switching based on epsilon-near-zero multilayer films," Scientific Reports, vol. 6, Article ID 22941, 2016.

[11] C. L. Wu, Y. H. Lin, S. P. Su et al., "Enhancing optical nonlinearity in non-Stoichiometric SiN waveguide for cross-wavelength all-optical data processing," Acs Photonics, vol. 2, no. 8, pp. 11411154, 2015.

[12] G. Priem, P. Dumon, W. Bogaerts, D. Van Thourhout, G. Morthier, and R. Baets, "Optical bistability and pulsating behaviour in Silicon-On-Insulator ring resonator structures," Optics Express, vol. 13, no. 23, pp. 9623-9628, 2005.

[13] M. R. Shcherbakov, S. Liu, V. V. Zubyuk et al., "Ultrafast all-optical tuning of direct-gap semiconductor metasurfaces," Nature Communications, vol. 8, no. 1, 2017.

[14] A. K. Azad, H. Chen, S. R. Kasarla et al., "Ultrafast optical control of terahertz surface plasmons in subwavelength hole arrays at room temperature," Applied Physics Letters, vol. 95, Article ID 011105, 2009.

[15] X. Fang, M. Lun Tseng, J. Ou, K. F. MacDonald, D. Ping Tsai, and N. I. Zheludev, "Ultrafast all-optical switching via coherent modulation of metamaterial absorption," Applied Physics Letters, vol. 104, no. 14, Article ID 141102, 2014.

[16] W. X. Lim, M. Manjappa, Y. K. Srivastava et al., "Ultrafast AllOptical Switching of Germanium-Based Flexible Metaphotonic Devices," Advanced Materials, vol. 30, no. 9, 2018.

[17] S. B. Choi, J. S. Kyoung, H. S. Kim et al., "Nanopattern enabled terahertz all-optical switching on vanadium dioxide thin film," Applied Physics Letters, vol. 98, no. 7, Article ID 071105, 2011.

[18] C. Rios, M. Stegmaier, P. Hosseini et al., "Integrated all-photonic non-volatile multi-level memory," Nature Photonics, vol. 9, no. 11, pp. 725-732, 2015.

[19] D. Tanaka, Y. Shoji, M. Kuwahara et al., "Ultra-small, selfholding, optical gate switch using Ge2Sb $2 \mathrm{Te} 5$ with a multimode Si waveguide," Optics Express, vol. 20, no. 9, pp. 1028310294, 2012.

[20] M. O. A. Ellis, E. E. Fullerton, and R. W. Chantrell, "All-optical switching in granular ferromagnets caused by magnetic circular dichroism," Scientific Reports, vol. 6, Article ID 30522, 2016.

[21] S. Gerlach, L. Oroszlany, D. Hinzke et al., "Modeling ultrafast all-optical switching in synthetic ferrimagnets," Physical Review B: Condensed Matter and Materials Physics, vol. 95, no. 22, Article ID 224435, 2017.

[22] X. Li, R. Liu, H. Xie et al., "Tri-phase all-optical switching and broadband nonlinear optical response in Bi2Se3 nanosheets," Optics Express, vol. 25, no. 15, pp. 18346-18354, 2017.

[23] Y. Wu, Q. Wu, F. Sun et al., "Emergence of electron coherence and two-color all-optical switching in MoS2 based on spatial self-phase modulation," Proceedings of the National Acadamy of Sciences of the United States of America, vol. 112, no. 38, pp. 11800-11805, 2015.

[24] C.-H. Lee, G.-H. Lee, A. M. Van Der Zande et al., "Atomically thin $\mathrm{p}-\mathrm{n}$ junctions with van der Waals heterointerfaces," Nature Nanotechnology, vol. 9, no. 9, pp. 676-681, 2014.

[25] C. Tan, X. Fu, Y. Hu et al., "Plasma optical modulation for lasers based on the plasma induced by femtosecond pulses," Optics Express, vol. 25, no. 13, pp. 14065-14076, 2017. 
[26] C. Tan, Q. Wang, and X. Fu, “Topological insulator Sb2Te3 as an optical media for the generation of ring-shaped beams," Optical Materials Express, vol. 4, no. 10, pp. 2016-2025, 2014.

[27] Y. Tang and X. Fu, "Evolution of low power laser affected by the plasma with gaussian profile," in Energy Science and Applied Technology: Proceedings of the 2nd International Conference on Energy Science and Applied Technology, pp. 321-324, CRC Press, 2015. 

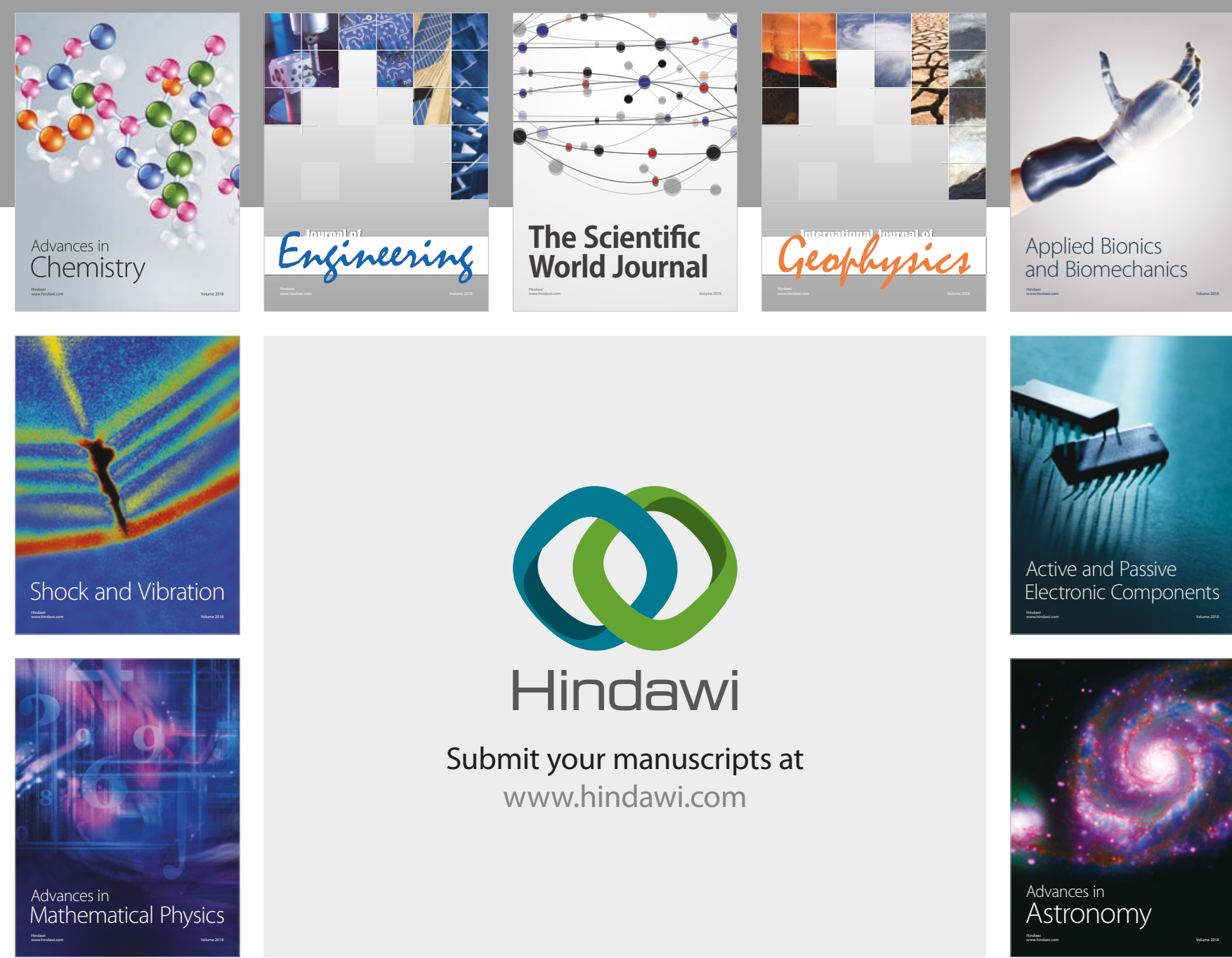

Submit your manuscripts at

www.hindawi.com

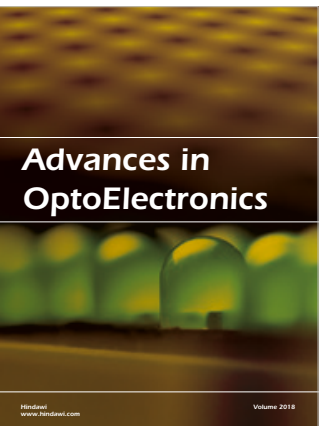

\section{Rotcting Machinery}
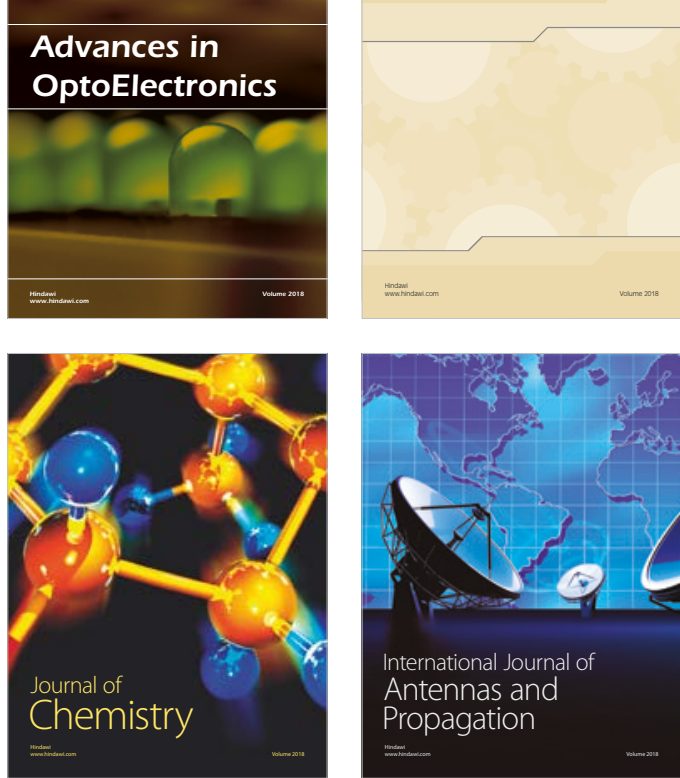

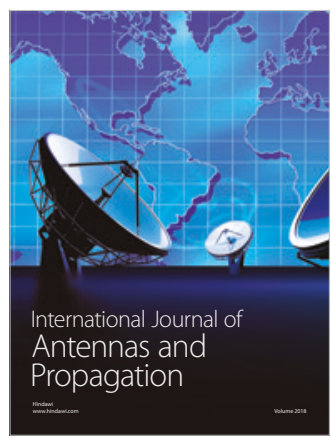

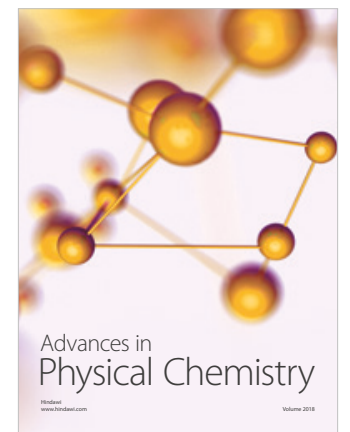

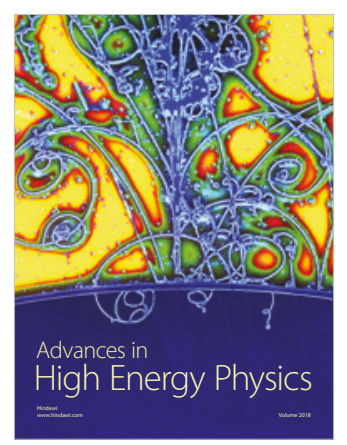

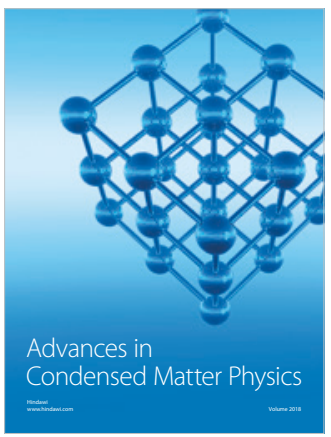

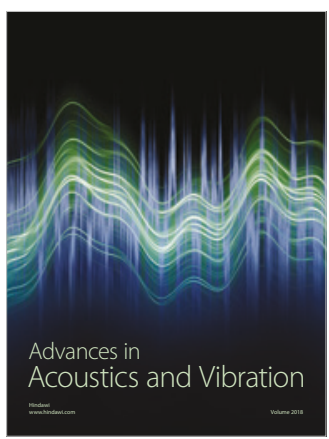

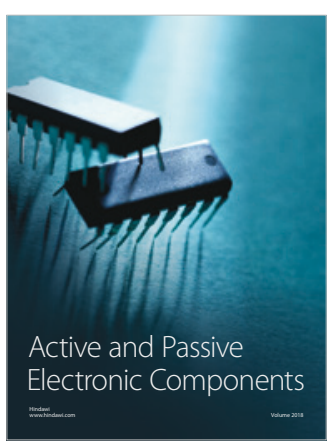
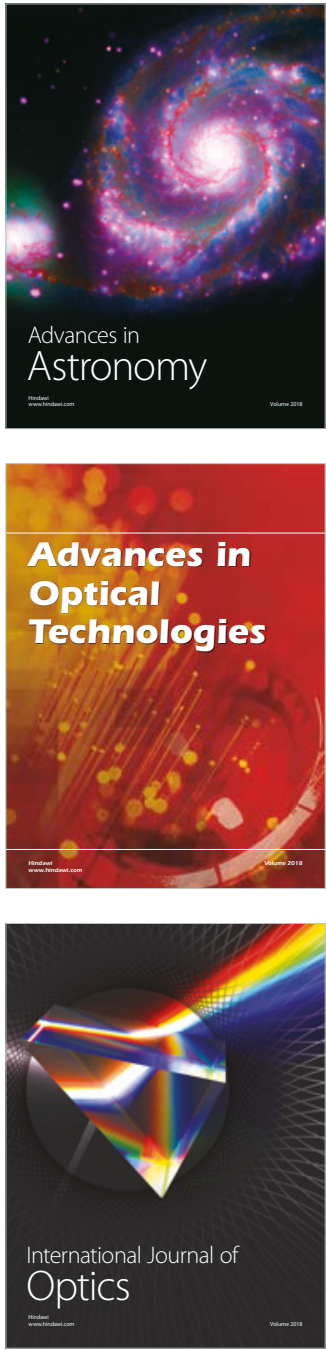